\title{
Nuclear Tensor Force and Effective Pions in the Relativistic Hartree-Fock Formalism
}

\author{
S. Marcos ${ }^{1, a}$, M. López-Quelle ${ }^{1}$, R. Niembro1 ${ }^{1}$, and L.N. Savushkin² \\ ${ }^{1}$ University of Cantabria, E-39005 Santander, Spain \\ ${ }^{2}$ St. Petersburg University for Telecommunications, 191065 St. Petersburg, Russia
}

\begin{abstract}
.
In the framework of nonlinear nuclear models based on the relativistic Hartree-Fock approximation, we have isolated the contribution of the tensor force of pions in the effective $\mathrm{NN}$ interaction, by means of two different approximate methods, recently developed by us, in order to dilucidate its role in a variety of nuclear properties. Results show that a reduction of the contribution of this tensor force considerably influences the spin-orbit splittings and magic gaps in the spin-unsaturated midweight ${ }^{56} \mathrm{Ni}$ nucleus as well as the behaviour of the total binding energies with $\mathrm{A}$ in heavy nuclei. Both methods give similar results. We also study the evolution of the splitting of the proton $1 d$ spin-orbit doublet in the chain $Z=14$, from $N=20$ to $N=28$, and the neutron $2 p-1 f$ shell in the chain $N=28$, from the ${ }^{48} \mathrm{Ca}$ nucleus to the ${ }^{42} \mathrm{Si}$ nucleus. Whereas, in the first case, the pion tensor force (PTF) plays an important role and its reduction is needed to reproduce the corresponding experimental results; in the second case, the quenching of the neutron $2 p_{3 / 2}-1 f_{7 / 2}$ gap in the mentioned isotonic chain $N=28$ is hardly affected by the PTF.
\end{abstract}

\section{Introduction}

Recently, in the framework of nonlinear nuclear models based on the relativistic Hartree-Fock approximation (RHFA), that include pions with a pseudovector coupling for the $\pi$-nucleon interaction, we have developed two ways to identify the pion tensor force (PTF) contribution [1,2]. On one way [1], we have observed that in the limit of the pion mass $m_{\pi} \rightarrow 0$, the finite range part of the NN interaction generated by pions contributes to the energy only through the PTF. This enables us to determine, in an approximate way, the main contribution of this force to the energy. On the other way [2], in the RHFA, in the expectation value of the Hamiltonian, the pion exchange contribution appears as a diagonal matrix where the PTF shows up and enables us to calculate directly its contribution to the energy.

Due to the strong strength of the PTF at very short distances between nucleons, one can expect a considerable weakening of the effect of this force due to short-range NN correlations. As the RHFA does not contain this kind of correlations, we think it is justified to reduce by hand the PTF contribution to the energy as done in $[1,2]$. As a result, the single-particle $(s p)$ spectra in spin-unsaturated heavy nuclei [1] and the total binding energy of certain isotopic families [2] improve considerably.

\footnotetext{
a e-mail: marcoss@unican.es
} 
In this work, using the models developed in [1,2], firstly, we study the influence of the PTF on the $s p$ level structure of the midweight doubly magic ${ }^{56} \mathrm{Ni}$ nucleus and the total binding energy of the lead isotopic chain and compare the results obtained with both methods. Recently, after appearance of new experimental data, the attention has been paid to the evolution of the splitting of the proton $1 d$ spin-orbit (SO) doublet in the isotopic chain $Z=14$ when $N$ increases from $N=20$ to $N=28$ and to the attenuation of the neutron gap $2 p_{3 / 2}-1 f_{7 / 2}$ along the isotonic chain $N=28$ when $Z$ decreases from $Z=20$ to $Z=14$ [3-5]. In the present work, we apply our models to study the tensor force effect in these two problems.

\section{Models}

We write the effective Lagrangian density $\mathcal{L}$ as [1],

$$
\mathcal{L}=\mathcal{L}_{0}\left(\varphi, \sigma, \omega_{\mu}, \boldsymbol{\rho}_{\mu}, \boldsymbol{\pi}, A_{\mu}\right)+\mathcal{L}_{\text {int }}(\text { meson-nucleon })-U_{\mathrm{NL}}(\text { meson-meson }),
$$

where $\mathcal{L}_{0}$ describes the free system and is given in [1],

$$
\mathcal{L}_{\text {int }}=-g_{\sigma} \bar{\varphi} \sigma \varphi-g_{\omega} \bar{\varphi} \gamma^{\mu} \omega_{\mu} \varphi-g_{\rho} \bar{\varphi} \gamma^{\mu} \rho_{\mu} \cdot \tau \varphi+\frac{f_{\pi}}{m_{\pi}} \bar{\varphi} \gamma_{5} \gamma^{\mu} \partial_{\mu} \pi \cdot \tau \varphi-\mathrm{e} \bar{\varphi} \gamma^{\mu} \frac{1+\tau_{3}}{2} A_{\mu} \varphi
$$

represents the interaction of nucleons with the different meson fields (we have not taken into account the $N \omega$ and $N \rho$ tensor couplings), and the nonlinear piece

$$
U_{\mathrm{NL}}=\frac{1}{3} \bar{b} M\left(g_{\sigma} \sigma\right)^{3}+\frac{1}{4} \bar{c}\left(g_{\sigma} \sigma\right)^{4}
$$

takes into account self-interactions of the $\sigma$ meson. $\varphi, \sigma, \omega_{\mu}, \boldsymbol{\rho}_{\mu}$ and $\boldsymbol{\pi}$ denote the field operators $\left(\boldsymbol{\rho}_{\mu}\right.$ and $\pi$ are vectors in the isospin space) corresponding to nucleons and to the $\sigma, \omega, \rho$ and $\pi$ mesons, respectively. Their corresponding free masses are denoted by the symbols $M, m_{\sigma}, m_{\omega}, m_{\rho}$ and $m_{\pi}$. $A_{\mu}$ is the electromagnetic field, involving the Coulomb interaction between protons. The components of the vector $\tau$ in the isospin space are the three standard Pauli matrices. $\bar{b}$ and $\bar{c}$ are dimensionless parameters.

We consider some models given in [1, 2] where we have used the physical masses $M=939 \mathrm{MeV}$, $m_{\omega}=783 \mathrm{MeV}, m_{\rho}=770 \mathrm{MeV}$ and $m_{\pi}=138 \mathrm{MeV}$. We have chosen $g_{\rho}$ to get a suitable symmetry energy. The values of the parameters of the models are given in Table 1. Models are labelled here in a more simple way than in $[1,2]$. The notation $P V$ stands for the pseudovector coupling we have taken for the $\pi N$ vertex.

Table 1. Values of the free fitting parameters $\left(m_{\sigma}, g_{\sigma}, g_{\omega}, g_{\rho}, \bar{b}, \bar{c}\right)$ used in our models. For the $\pi$ mesons, we have taken $f_{\pi}^{2} / 4 \pi \simeq 0.076$, corresponding to the $\pi N$ pseudoscalar coupling $g_{\pi}^{2} / 4 \pi=14$, in the range of experimental data. Model $P V a$ includes the entire PTF contribution. In models $P V e$ and $P V e^{\prime}$, the PTF contribution is removed using the approximate method developed in [1] and [2], respectively. In model $P V c^{\prime}$, about $1 / 3$ of the PTF contribution is kept [2].

\begin{tabular}{l|c|c|c|c|c|c|}
\hline Model & $m_{\sigma}, \mathrm{MeV}$ & $g_{\sigma}^{2} / 4 \pi$ & $g_{\omega}^{2} / 4 \pi$ & $g_{\rho}^{2} / 4 \pi$ & $\bar{b} \times 10^{3}$ & $\bar{c} \times 10^{3}$ \\
\hline$P V a$ & 443.28 & 5.3215 & 10.393 & 0.72 & -4.361 & -7.257 \\
$P V e$ & 441.57 & 4.9822 & 9.3441 & 0.62 & -5.497 & -9.308 \\
$P V e^{\prime}$ & 441.72 & 5.0154 & 9.5096 & 0.67 & -5.260 & -8.948 \\
$P V c^{\prime}$ & 441.85 & 5.0898 & 9.7595 & 0.69 & -4.970 & -8.366 \\
\hline
\end{tabular}

In calculations, we assume a spherical symmetry description of the nuclei considered. Pairing correlations are treated in the BCS approximation [6]. The pairing intensities for neutrons and protons 
were chosen so that they provide a well suited approximation for the pairing gap and binding energy simultaneously for the nucleus considered (experimental data are taken from [7]). The BCS equations have been solved self-consistently within the RHFA, so that the pairing gap and binding energy come up from the calculation.

\section{Results}

The neutron $s p$ energy levels of the neutron and proton spin-unsaturated ${ }^{56} \mathrm{Ni}$ nucleus are given in Fig. 1a) for parametrizations of Table 1. The proton spectrum behaves in a similar way as the neutron spectrum does. We observe that the weakening of the PTF contribution affects the SO splittings in the expected way [1]. Model $P V c^{\prime}$, keeping about $1 / 3$ of the PTF, gives a better result for heavy nuclei [2] than for the ${ }^{56} \mathrm{Ni}$ nucleus, which requires somewhat more PTF contribution to fit the experimental $v 1 f$ SO splitting and the $N=28$ gap. The tensor force effect on the $v 1 p$ and $v 1 d$ SO splittings is strong but there is no experimental data to be compared with. The results of models $P V e$ and $P V e^{\prime}$ show that both methods to isolate the tensor part give similar results.

Figure 1. a) The level spectrum $E_{i}$ of the ${ }^{56} \mathrm{Ni}$ nucleus using the models given in Table 1. b) The binding energy per particle $E / A$ of the isotopic chain of the ${ }^{208} \mathrm{~Pb}$ nucleus.


Fig. 1b) shows the binding energy per particle for the isotopic chain of the ${ }^{208} \mathrm{~Pb}$ nucleus for models of Table 1. A good agreement of model $P V c^{\prime}$ with the experimental data is reached.

In Fig. 2a), we show the results obtained with the models of Table 1 to give an idea of the influence of the PTF contribution on the evolution of the $\pi 1 d$ SO splitting when going from the ${ }^{34} \mathrm{Si}$ nucleus to the ${ }^{42} \mathrm{Si}$ nucleus by adding $1 f_{7 / 2}$ neutrons. To consider a spherical symmetry description for some of the nuclei studied is, certainly, a strong assumption (see a discussion on the shape of the ${ }^{42} \mathrm{Si}$ nucleus in [8] ) but it allows us to more easily gain insight into the role of the separate tensor part of the relativistic mean field. The experimental attenuation of $1.94 \mathrm{MeV}$ [8] of the $1 d \mathrm{SO}$ splitting along the $Z=14$ isotopic chain depicted in Fig. 2 a) is very approximately provided by model $P V c^{\prime}$.

A shell model analysis of the data has revealed that the traditional $N=28$ shell gap in the nuclear $s p$ spectrum is eroded when moving away from the stability line [8,9]. In particular, when $Z$ decreases from $Z=20$ to $Z=14$, the gradual removal of protons from the $\pi 2 s_{1 / 2}$ and $\pi 1 d_{3 / 2}$ orbitals reduces the size of the gap by about $1 \mathrm{MeV}$ [10], $330 \mathrm{keV}$ per pair of protons. Some explanation has been given in $[4,5]$. In Fig. 2b), we present the evolution of the $N=28$ gap $\left(2 p_{3 / 2}-1 f_{7 / 2}\right)$ for the models considered 
Figure 2. a) The proton $1 d$ spin-orbit splitting for $Z=14$ from $N=20$ to $N=28$. b) The neutron $2 p_{3 / 2}-1 f_{7 / 2}$ gap from the ${ }^{48} \mathrm{Ca}$ nucleus toward the ${ }^{42} \mathrm{Si}$ nucleus using the models given in Table 1 .
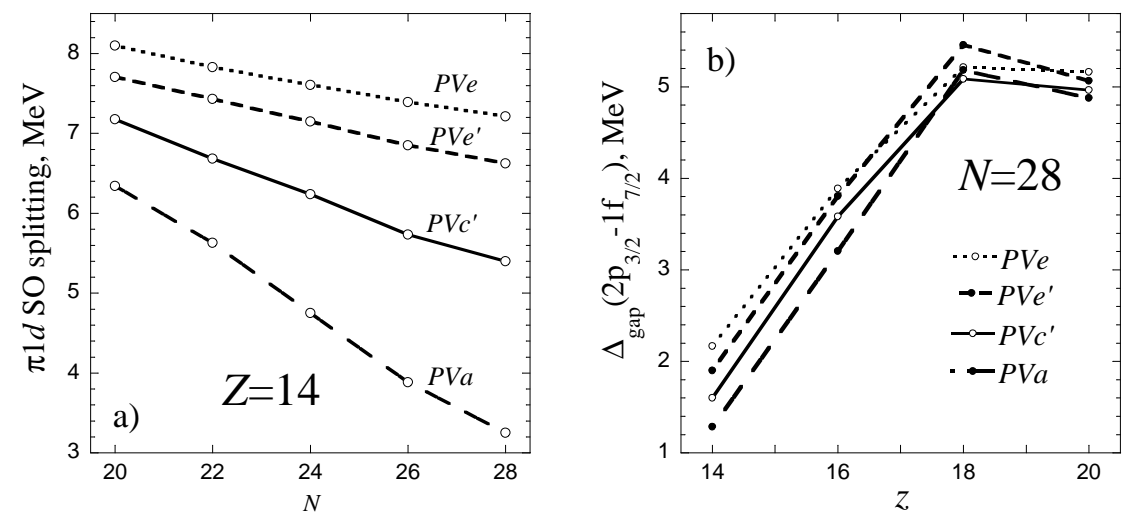

here. The tendency is similar in all models. The two different slopes of the curves depicted around $Z=18$ correspond to the evacuation of the orbital $2 s_{1 / 2}$ on the right and the orbital $1 d_{3 / 2}$ on the left. In all cases, the quenching of the gap is considerably larger than the experimental one. The lack of the PTF contribution only decreases the excessive gap attenuation in about $0.5 \mathrm{MeV}$, which means that the PTF contribution has a meager influence on the evolution of this gap.

\section{Conclusions}

In summary, in the RHFA, we have shown the influence of the PTF contribution on the $s p$ spectrum of the ${ }^{56} \mathrm{Ni}$ nucleus and the binding energy per particle of the isotopic chain of the lead. An improvement in the description of both properties can be achieved by a reduction of the PTF contribution. The best results correspond to keep about one third of this contribution. We also have investigated the influence of the PTF in both the evolution of the $\pi 1 d$ SO splitting along the $Z=14(N=20 \ldots .28)$ isotopic chain and the evolution of the $v\left(2 p_{3 / 2}-1 f_{7 / 2}\right)$ gap along the $N=28(Z=14 \ldots 20)$ isotonic chain. Whereas the first mechanism is very sensitive to the PTF, the second one is hardly dependent on the PTF. We almost reproduce the experimental $\pi 1 d$ SO splitting attenuation with one third of the PTF.

\section{References}

[1] S. Marcos, M. López-Quelle, R. Niembro and L. N. Savushkin, Yad. Fiz. 76, 604 (2013) [Phys. Atom. Nucl. 76, 562 (2013)].

[2] S. Marcos, M. López-Quelle, R. Niembro and L. N. Savushkin, Yad. Fiz. (2013) (in press).

[3] D. Tarpanov, H. Liang, N. Van Giai, and C. Stoyanov, Phys. Rev. C 77, 054316 (2008).

[4] F. Nowacki and A. Poves, Phys. Rev. C 79, 014310 (2009).

[5] K. Kaneko, Y. Sun, T. Mizusaki and M. Hasegawa, Phys. Rev. C 83, 014320 (2011).

[6] D. M. Brink and R. A. Broglia, Nuclear Superfluidity (Cambridge University Press, United Kingdom, 2005).

[7] T-2 Nuclear Information Service. Los Alamos National Laboratory, http://t2.lanl.gov/.

[8] B. Bastin et al., Phys. Rev. Lett. 99, 022503 (2007).

[9] L. Gaudefroy et al., Phys. Rev. Lett. 102, 092501 (2009).

[10] L. Gaudefroy et al., Phys. Rev. Lett. 97, 092501 (2006). 